\title{
Amplifier Test
}

\author{
J. M. Cesaratto
}

Jan. 17, 2013

\section{Purpose}

Test the signal level at the load of the back end chain. Compare signal levels for a burst type signal (like that of the excitation system) versus a continuous sinusoidal signal.

\section{Experimental Procedure}

For this test, we used the existing back end configuration as shown in Figure ??. For the burst type signal we used the excitation system, and for the continuous type signal we used an arbitrary function generator. The frequencies of the signal are 100, 200, 300, $400 \mathrm{MHz}$. The signals were adjusted to be approximately the same level when input into the back end chain. At the MUX out load we measure the signal with a Tektronix oscilloscope, triggering on the load signal itself, recording $1 \mathrm{~K}$ turns (fiducial triggers). The negative $\left(\mathrm{V}_{\text {load }}^{-p}\right)$ and positive $\left(\mathrm{V}_{\text {load }}^{p}\right)$ peak voltages generated were recorded, from which peak to peak voltages $\left(\mathrm{V}_{\text {load }}^{\text {pp }}\right)$ were computed. The input signal was attenuated by $10 \mathrm{~dB}$ compared to the normal operating signal used for beam excitation as a cautionary measure. One measurement was made at normal operating power. We measure the loads of two amplifiers, amp 2 and amp 4.

\section{Measurement}

Tables 1 and 2 display the input signals and measured signals for the different frequencies when monitoring the load of amplifier 4 and amplifier 2 , respectively.

Operating at the nominal input signal, removed additional $10 \mathrm{~dB}$ attenuator $(-11 \mathrm{dBm}$ signal level at $100 \mathrm{MHz}$ at entrance of Wideband Gate). Burst: $\mathrm{V}_{\text {load }}^{-p}=-78 \mathrm{mV}, \mathrm{V}_{\text {load }}^{p}=58 \mathrm{mV}, \mathrm{V}_{\text {load }}^{p p}=136 \mathrm{mV}$. Continuous: Burst: $\mathrm{V}_{\text {load }}^{-p}=-44 \mathrm{mV}, \mathrm{V}_{\text {load }}^{p}=49 \mathrm{mV}, \mathrm{V}_{\text {load }}^{\text {po }}=93 \mathrm{mV}$

\section{Conclusions}

It seems that the burst and continuous signals are rather similar in levels measured at the load, contrary to our initial thought that the continuous levels were about twice that of the burst levels (2:1). See .wfm files listed for actual signals recorded for $1 \mathrm{~K}$ turns (fiducial triggers). 
Table 1: Amplifier 4 load measurement with corresponding input signal levels for burst and continuous type signals

\begin{tabular}{|c|c|c|c|c|c|}
\hline $\begin{array}{c}\text { File } \\
\left(130117_{-}\right)\end{array}$ & $\begin{array}{c}\text { Frequency } \\
(\mathrm{MHz})\end{array}$ & $\mathrm{V}_{i n}^{p p}$ & $\begin{array}{l}\mathrm{V}_{\text {load }}^{-p} \\
(\mathrm{~m}\end{array}$ & $\begin{array}{l}\mathrm{V}_{\text {load }}^{p} \\
V)\end{array}$ & $\mathrm{V}_{\text {load }}^{p p}$ \\
\hline \multicolumn{6}{|c|}{ Burst } \\
\hline & 100 & 53.0 & -32.0 & 40.8 & 72.8 \\
\hline & 200 & 50.0 & -26.0 & 25.0 & 51.0 \\
\hline 145128 & 300 & 45.7 & -8.8 & 17.2 & 26.0 \\
\hline 151339 & 400 & 40.5 & -6.6 & 13.6 & 20.2 \\
\hline \multicolumn{6}{|c|}{ Continuous } \\
\hline & 100 & 56.0 & -45.2 & 45.2 & 90.4 \\
\hline & 200 & 49.9 & -21.6 & 21.6 & 43.2 \\
\hline & 300 & 45.8 & -13.8 & 13.8 & 27.6 \\
\hline 151918 & 400 & 40.7 & -9.6 & 10.2 & 19.8 \\
\hline
\end{tabular}

Table 2: Amplifier 2 load measurement with corresponding input signal levels for burst and continuous type signals

\begin{tabular}{cccccc}
\hline $\begin{array}{c}\text { File } \\
(130117+)\end{array}$ & $\begin{array}{c}\text { Frequency } \\
(\mathrm{MHz})\end{array}$ & $\mathrm{V}_{\text {in }}^{p p}$ & $\begin{array}{c}\mathrm{V}_{\text {load }}^{-p} \\
(\mathrm{mV})\end{array}$ & \begin{tabular}{c}
$\mathrm{V}_{\text {load }}^{p}$ \\
\hline \multicolumn{7}{c}{ Burst }
\end{tabular} & $\mathrm{V}_{\text {load }}^{p p}$ \\
\hline 153419 & 100 & 53.0 & -52.0 & 39.6 & 91.6 \\
153537 & 200 & 50.0 & -21.2 & 23.0 & 44.2 \\
153729 & 300 & 45.7 & -12.6 & 5.0 & 17.2 \\
153947 & 400 & 40.5 & -10.2 & 5.0 & 15.2 \\
\hline \multicolumn{6}{c}{ Continuous } \\
\hline 152910 & 100 & 56.0 & -42.8 & 43.6 & 86.4 \\
152805 & 200 & 49.9 & -18.4 & 18.6 & 37.0 \\
152521 & 300 & 45.8 & -8.6 & 8.8 & 17.4 \\
152302 & 400 & 40.7 & -6.6 & 6.2 & 12.8 \\
\hline \hline
\end{tabular}

